\begin{tabular}{|c|l|}
\hline Title & Inelastic collision processes of low energy protons in liquid water \\
\hline Author(s) & Date, H.; Sutherland, K.L.; Hay ashi, T.; Matsuzaki, Y.; Kiy anagi, Y. \\
\hline Citation & $\begin{array}{l}\text { Radiation Physics and Chemistry, 75(2), 179-187 } \\
\text { https://doi.org/10.1016/.radphyschem.2005.10.002 }\end{array}$ \\
\hline Issue Date & 2006-02 \\
\hline Doc URL & http://hdl.handle.net/2115/7380 \\
\hline Type & article(author version) \\
\hline File Information & RPC75_2.pdf \\
\hline
\end{tabular}

Instructions for use 


\title{
Inelastic collision processes of low energy protons in liquid water
}

\author{
H. Date*, K.L. Sutherland ${ }^{1}$, T. Hayashi ${ }^{1}$, Y. Matsuzaki ${ }^{2}$, Y. Kiyanagi ${ }^{2}$ \\ School of Medicine, Hokkaido University, Sapporo 060-0812, Japan \\ ${ }^{1}$ Japan Science and Technology Agency, Tokyo 105-6218, Japan \\ ${ }^{2}$ Graduate School of Engineering, Hokkaido University, Sapporo 060-8628, Japan
}

\begin{abstract}
Production probabilities of ion and excited particle species along the proton beam track in liquid water are estimated around the Bragg peak region, taking into account charge changing processes and energetic secondary electron ( $\delta$-ray) behavior. Ionization and excitation processes are divided into two categories in this study: primary processes associated with direct proton (or hydrogen) interaction and secondary processes arising from the electrons ejected by the primary process. We show that the number of events in the secondary processes producing ions and excited particles is larger than that of the primary processes around the Bragg peak while neutralized protons (i.e., hydrogen) with low energy have a large contribution to direct ionization. Effects of charge changing processes on ionization and excitation are also discussed.
\end{abstract}

Key words: proton Bragg peak, inelastic collision, $\delta$-ray, charge transfer, cross sections, liquid water

*Corresponding author:

Department of Health Sciences, School of Medicine, Hokkaido University

N12-W5, Kitaku, Sapporo 060-0812, JAPAN

Phone\#: +81-11-706-3423

Fax\#: +81-11-706-4916

E-mail: date@cme.hokudai.ac.jp 


\section{Introduction}

When radiation particles interact with bio-materials, the particles transfer most of their energy to electrons. The resultant transformation of atoms and molecules in the material occurs through both electron collisions and direct interaction with primary radiation particles (incident protons). The behavior of proton beams in material (specifically water) can be viewed as a canonical system in which incident protons and secondary electrons interact with bio-materials (Fig.1). A proton transfers a maximum of about $4 \mathrm{~m}_{\mathrm{e}} / \mathrm{m}_{\mathrm{p}}\left(\mathrm{m}_{\mathrm{p}}\right.$ : the proton mass, $\mathrm{m}_{\mathrm{e}}$ : the electron mass) of its energy to electrons through an ionization collision. With such a large energy share, electrons generated through proton induced direct ionization with water molecules have, therefore, sufficient energy and probability to induce further ionization and excitation processes with ambient molecules. The energetic secondary electron is the so-called $\delta$-ray.

In the energy range of interest, protons travel in essentially a straight line, loosing energy through collisions. At the Bragg peak region of the proton track, low energy protons may be neutralized through the capture of electrons from water molecules. Conversely, hydrogen atoms arising from this neutralization process may also undergo electron loss (electron stripping) as well. Both processes must be balanced in a dynamic equilibrium state. These charge changing processes take place along the proton's original straight trajectory. The tracks of the produced electrons spread out in a tree-branch structure (see Fig.1). Collision interaction products (ions and/or excited particle species) are also generated along the track within the branch structure. Although the density is lower, the number of the products is higher in the outer region than around the original proton track due to the secondary electron processes.

Conventionally, the relative biological effectiveness (RBE) of radiation has been discussed based upon the energy deposit per unit length, that is, the stopping power ( $\mathrm{dE} / \mathrm{dx})$ or linear energy transfer (LET). However, from a microscopic point of view, the biological-cell mutation probability depends on the ionization and/or excitation rate, not the energy deposit itself. 
Accordingly, the production rate of ionization and excitation particle species per unit volume in the track structure is essential for determining the radiation weighting factor $\left(\mathrm{w}_{\mathrm{R}}\right)$ or quality factor of radiation $(\mathrm{Q})$.

In this study, we calculate the probability of ionization and excitation events by a proton incident in liquid water, in which secondary electron and charge transfer processes are also important. By taking into account event occurrences for both ionization and excitation by secondary electrons and charge changing reaction processes, we estimate the effective production rate of ionization and excitation around the Bragg peak region per unit traveled of the incident proton. We also investigate implicit effects of the secondary electrons on the stopping power of protons.

\section{Method of calculation}

In the present analysis, we estimate event probabilities for ionization and excitation of water molecules. Ionization and excitation occur in the course of proton transport and in subordinate processes associated with charge change and electron transport.

Charge changing processes have an important role in proton energy degradation, particularly around the Bragg peak region (e.g., Rudd et al., 1992, Uehara et al., 2000). Low energy protons may capture electrons from water molecules becoming neutral-hydrogen atoms. Electrons may also be stripped from a hydrogen atom. In a dynamic charge equilibrium state, the probabilities of both processes are given by

$\Phi_{0}=\frac{\sigma_{10}}{\sigma_{01}+\sigma_{10}}$, and $\Phi_{1}=\frac{\sigma_{01}}{\sigma_{01}+\sigma_{10}}$

where $\sigma_{10}$ and $\sigma_{01}$ are the cross sections for

$\mathrm{p}+\mathrm{H}_{2} \mathrm{O} \rightarrow \mathrm{H}+\mathrm{H}_{2} \mathrm{O}^{+}$(electron capture)

and 
$\mathrm{H}+\mathrm{H}_{2} \mathrm{O} \rightarrow \mathrm{p}+\mathrm{e}+\mathrm{H}_{2} \mathrm{O}$ (electron loss).

For an incident proton beam, the effective cross sections for ionization and excitation can be described by

$\sigma_{\text {ion }}=\sigma_{\text {ion }}^{(\mathrm{H})} \Phi_{0}+\sigma_{\text {ion }}^{(\mathrm{p})} \Phi_{1}$

and

$\sigma_{\mathrm{exc}}=\sigma_{\mathrm{exc}}^{(\mathrm{H})} \Phi_{0}+\sigma_{\mathrm{exc}}^{(\mathrm{p})} \Phi_{1}$

where $\sigma_{\text {ion }}^{(\mathrm{H})}, \sigma_{\text {ion }}^{(\mathrm{p})}$ and $\sigma_{\mathrm{exc}}^{(\mathrm{H})}, \sigma_{\mathrm{exc}}^{(\mathrm{p})}$ are ionization and excitation cross sections for hydrogen $(\mathrm{H})$ and proton $(\mathrm{p})$, respectively.

Some electrons generated by the ionization in Eq.(2) are energetic and have the ability to ionize and excite other water molecules. However, the secondary electron energy after primary ionization is below a few thousands eV. Accordingly, most of the electrons can move only less than 0.1-1 $\mu \mathrm{m}$ in CSDA range (e.g., Atomic Data, 1972). Hereafter, we refer to these processes by electrons (namely $\delta$-rays) "secondary processes", while we refer to the direct ionization and excitation by protons or hydrogen atoms "primary processes". The secondary processes are dominated by the energy spectra of electrons ejected in the primary ionization. The probable energy distribution of the electrons is represented by the single differential cross section $\mathrm{d} \sigma / \mathrm{dE}$ (differential in energy transfer E),

$\frac{d \sigma_{\text {ion }}}{d E}=\sum_{\text {all } j} G_{j} \frac{d \sigma_{\text {ion }}^{j}}{d W_{j}}$,

where $\mathrm{W}_{\mathrm{j}}=\mathrm{E}-\mathrm{I}_{\mathrm{j}}$ is the secondary electron kinetic energy, $\mathrm{I}_{\mathrm{j}}$ is the ionization energy of sub-shell $\mathrm{j}$ in liquid water and $\mathrm{G}_{\mathrm{j}}$ is the partitioning factor (Rudd et al., 1992 and Dingfelder el al., 2000). If we consider the event counts for ionization and excitation in the electron processes from the initial energy $W$ (just after the ejection) down to the cutoff energy, as $k_{\text {ion }}(W)$ and $k_{\text {exc }}(W)$, the 
effective cross sections for ionization and excitation by secondary processes are given by $\sigma_{\text {ion }}^{\text {elec }}=\int_{\mathrm{W}_{\text {min,ion }}}^{\infty} \frac{\mathrm{d} \sigma_{\text {ion }}}{\mathrm{dE}} \mathrm{k}_{\text {ion }}(\mathrm{W}) \mathrm{dW}$

and

$\sigma_{\mathrm{exc}}^{\mathrm{elec}}=\int_{\mathrm{W}_{\text {min,exc }}}^{\infty} \frac{\mathrm{d} \sigma_{\mathrm{ion}}}{\mathrm{dE}} \mathrm{k}_{\mathrm{exc}}(\mathrm{W}) \mathrm{dW}$,

where $\mathrm{W}_{\text {min,ion }}$ and $\mathrm{W}_{\text {min,exc }}$ are cutoff energies equivalent to the ionization and excitation thresholds for the electron impact. It should be noted that the relation $\frac{d \sigma_{\text {ion }}(E)}{d E}=\frac{d \sigma_{\text {ion }}(W)}{d W}$ is sustained for the transfer energy $\mathrm{E}$ and the ejected electron energy $\mathrm{W}$. Thus, the total cross sections for ionization and excitation including secondary electron processes become

$\sigma_{\text {ion }}^{\mathrm{p}+\mathrm{s}}=\left(\sigma_{\text {ion }}^{(\mathrm{H})}+\sigma_{\text {ion }}^{(\mathrm{H})-\mathrm{elec}}\right) \Phi_{0}+\left(\sigma_{\text {ion }}^{(\mathrm{p})}+\sigma_{\text {ion }}^{(\mathrm{p})-\text { elec }}\right) \Phi_{1}$

and

$\sigma_{\mathrm{exc}}^{\mathrm{p}+\mathrm{s}}=\left(\sigma_{\mathrm{exc}}^{(\mathrm{H})}+\sigma_{\mathrm{exc}}^{(\mathrm{H})-\mathrm{elec}}\right) \Phi_{0}+\left(\sigma_{\mathrm{exc}}^{(\mathrm{p})}+\sigma_{\mathrm{exc}}^{(\mathrm{p})-\mathrm{elec}}\right) \Phi_{1}$.

Here, $\sigma_{\mathrm{J}}^{(\mathrm{K})}$ denotes the cross section for process-J (ionization or excitation) by the primary ionization $\mathrm{K}$ (proton or hydrogen), and $\sigma_{\mathrm{J}}^{(\mathrm{K}) \text {-elec }}$ the cross section associated with electrons generated by the primary ionization $\mathrm{K}$ (proton or hydrogen). These are corresponding to Eqs.(5) and (6).

Charge changing processes may also induce the ionization and excitation of water molecules. In the electron loss process, an electron departs from hydrogen and travels with the same speed as the proton. That is to say, it has the kinetic energy $\mathrm{T} / \lambda$, where $\mathrm{T}$ is the proton energy and $\lambda=\mathrm{m}_{\mathrm{p}} / \mathrm{m}_{\mathrm{e}}=1836\left(\mathrm{~m}_{\mathrm{p}}\right.$ : the proton mass, $\mathrm{m}_{\mathrm{e}}$ : the electron mass). This electron may cause ionization and excitation as well as the secondary electron mentioned above. Moreover, an ion $\left(\mathrm{H}_{2} \mathrm{O}^{+}\right)$is produced in the electron capture process, which can also be regarded as an ionization event. The equivalent ionization and excitation cross sections by the charge changing reaction 
are given as:

$$
\sigma_{\text {ion }}^{\mathrm{CC}}=\sigma_{01} \Phi_{0} \mathrm{k}_{\text {ion }}(\mathrm{T} / \lambda)+\sigma_{10} \Phi_{1}
$$

and

$$
\sigma_{\mathrm{exc}}^{\mathrm{CC}}=\sigma_{01} \Phi_{0} \mathrm{k}_{\mathrm{exc}}(\mathrm{T} / \lambda)
$$

These cross sections are added to Eq.(7) and Eq.(8), respectively, to obtain a complete form of the effective total cross sections as a function of the incident particle (proton or hydrogen) energy. Finally, we can express them in event counts per unit length:

$$
\mathrm{N} \sigma_{\text {ion }}^{\text {total }}=\mathrm{N}\left(\sigma_{\text {ion }}^{(\mathrm{H})}+\sigma_{\text {ion }}^{(\mathrm{H}) \text {-elec }}+\sigma_{01} \mathrm{k}_{\text {ion }}(\mathrm{T} / \lambda)\right) \Phi_{0}+\mathrm{N}\left(\sigma_{\text {ion }}^{(\mathrm{p})}+\sigma_{\text {ion }}^{(\mathrm{p}) \text {-elec }}+\sigma_{10}\right) \Phi_{1}
$$

and

$$
\mathrm{N} \sigma_{\mathrm{exc}}^{\mathrm{total}}=\mathrm{N}\left(\sigma_{\mathrm{exc}}^{(\mathrm{H})}+\sigma_{\mathrm{exc}}^{(\mathrm{H})-\mathrm{elec}}+\sigma_{01} \mathrm{k}_{\mathrm{exc}}(\mathrm{T} / \lambda)\right) \Phi_{0}+\mathrm{N}\left(\sigma_{\mathrm{exc}}^{(\mathrm{p})}+\sigma_{\mathrm{exc}}^{(\mathrm{p})-\mathrm{elec}}\right) \Phi_{1}
$$

where $\mathrm{N}$ is the number of water molecules per unit volume.

\section{Collision cross sections}

\subsection{Proton and hydrogen}

For the impact cross sections of proton and hydrogen in liquid water, we adopt the semi-empirical model following Dingfelder et al. (2000) as their analytical cross sections lend themselves to the present analysis. For clarity, we present the cross-section here. For the excitation cross section for proton incident with energy $\tau$, the analytic form for a single excitation level $\mathrm{k}$ is given as

$$
\sigma_{\mathrm{exc}, \mathrm{k}}^{(\mathrm{p})}(\tau)=\frac{\sigma_{0}(\mathrm{Za})^{\Omega}\left(\tau-\mathrm{E}_{\mathrm{k}}\right)^{v}}{\mathrm{~J}^{\Omega+v}+\tau^{\Omega+v}}
$$

with sets of parameters for five levels in liquid water, a, J, v, and $\Omega$. Here, $\sigma_{0}$ is a constant $\left(=10^{-20} \mathrm{~m}^{2}\right), \mathrm{Z}$ the number of electrons in the target material, and $\mathrm{E}_{\mathrm{k}}$ the excitation energy. The excitation cross sections for neutral hydrogen impact are neglected in Dingfelder's paper, but 
we consider them the same as protons except the parameter $a$ of Eq.(13) which is assumed to be 3/4 of the proton value, following Miller and Green (1973).

The ionization cross section given by Dingfelder et al. (2000) is based upon the method by Rudd et al. (1992). The differential cross sections for five major ionization processes are in the analytical forms with several specific parameters similar to the excitation cross sections. We use the single differential cross section $\mathrm{d} \sigma / \mathrm{dE}$ in the summation formula (Eq.(38) in their paper) with the parameters for liquid water. The differential cross section for hydrogen is also presented by

$\left(\frac{\mathrm{d} \sigma}{\mathrm{dE}}\right)_{(\mathrm{H})}=\mathrm{g}(\tau)\left(\frac{\mathrm{d} \sigma}{\mathrm{dE}}\right)_{(\mathrm{p})}$

where, $g(\tau)=0.8\left(1+\exp \left[\frac{\log _{10}(\tau)-4.2}{0.5}\right]\right)^{-1}+0.9$, and subscripts $(H)$ and $(p)$ represent hydrogen and proton, respectively.

Analytic forms for charge-changing cross sections for electron capture and electron loss mentioned in the previous section are also taken from Dingfelder et al. (2000) and those curves fit well the other reported data (e.g., Toburen et al., 1968, Dagnac et al., 1970).

All of the cross sections given above are shown in Fig.1. The total ionization cross section in Fig.2 was obtained by integration of the single differential cross section $\mathrm{d} \sigma / \mathrm{dE}$ over electron energy, which duplicates correctly the one shown in the paper by Dingfelder et al. (2000).

\subsection{Electron}

In order to construct a Monte Carlo simulation of electrons generated by the primary ionization process through proton or hydrogen impact, three types of electron collisions need to be considered for water molecules in liquid state: elastic, ionization and excitation. Electron impact cross sections of water have been reported by many authors (e.g., IAEA-TECDOC-799). 
However, most of them are for water in vapor phase while the data for liquid water are scarce. Recently, some researchers have reported inelastic cross sections for liquid water, for example Emfietzoglou (2003) and Champion (2003). It seems to have been accepted that the ionization and excitation cross sections of water in liquid phase are significantly smaller than those in vapor phase especially for electron energy below $100 \mathrm{eV}$. In the present study, we adopt the cross sections for total ionization and total excitation by Emfietzoglou, which are in good agreement with those of earlier work by Pinblott et al. (1996) and Dingfelder et al. (1998). As for the elastic collision cross section, we use the experimental data in water vapor by Danjo and Nishimura (1985) with a multiplication factor 1.4. The multiplication factor has been chosen so as to fit the total cross section due to the paucity of the elastic cross section data. The elastic collision process is not so important in our calculation because the energy transfer in the elastic collision is minute and our aim is to focus on the inelastic event counts. The angular distribution of electrons after scattering is assumed to be constant ignoring angular anisotropy. We have confirmed that this assumption causes negligible differences in the present analysis.

The set of total ionization, total excitation and elastic cross sections is embedded into a Monte Carlo code. In order to determine the energy partition ratio of primary and secondary electrons after ionization, we use the algorithm by Grosswendt and Weibel (1978) with some fitting parameters by Green and Sawada (1972). The electron transport calculation is performed in the same manner as that of a previous paper by Date et al. (2003).

\section{Results and discussion}

Figure 3 shows stopping cross sections $\sigma_{\text {st }}(=\mathrm{S} / \mathrm{N}: \mathrm{S}$ is the stopping power, $\mathrm{N}$ the number of water molecules per unit volume) which were deduced from the impact cross sections given in Fig.2 to check the validity for constructing the conventional quantity. The stopping cross section demonstrates the Bragg peak well, and the shape is quite similar to ICRU-49 data 
(ICRU, 1993) except for that the peak value of $\sigma_{\text {st }}$ in the present result is about $5 \%$ larger than that of the ICRU liquid data.

The occurrence rates of ionization and excitation by electron impact as a function of initial electron energy are shown in Fig.4, which were obtained by the Monte Carlo simulation of electrons. The number in the vertical axis represents the average cumulative event frequency during the electron track motion from an initial energy E down to a cutoff energy (typically excitation threshold). Our results yield W-values in fairly good agreement with that of water vapor reported by Olivera et al. (1997).

Figure 5 shows the probable ionization counts per micrometer for the incident particle (proton or hydrogen), and Fig.6 the excitation counts. These were derived from the terms given in Eqs. (11) and (12). For example, the legend (p)-ion in Fig.5 represents $N \sigma_{\text {ion }}^{(\mathrm{p})} \Phi_{1}$ and (p)-e-ion is for $\mathrm{N} \sigma_{\mathrm{ion}}^{(\mathrm{p})-\mathrm{elec}} \Phi_{1}$, etc. Here, $\mathrm{N}$ is the density of water molecules $\left(=3.342 \times 10^{28} \mathrm{~m}^{-3}\right)$, and we reduced all results to $1 / \mu \mathrm{m}$ units. As shown in Fig.5, the direct ionization by protons occurs significantly in the energy region higher than $10^{4} \mathrm{eV}$ and the count of ionization by secondary electrons is larger than that of the direct proton ionization. Contrary to this, the direct ionization by hydrogen takes place mostly in the lower energy region $\left(<10^{5} \mathrm{eV}\right)$ and the contribution of ionization by electrons generated by hydrogen is minor. In Fig.6, we can see that the secondary electrons generated by protons are the dominant particles for the excitation process.

The event counts for charge changing processes are shown in Fig.7. It is recognized that the electron stripping process from hydrogen to proton results in a steep peak of the excitation probability by generated electrons between the hydrogen energy $10^{4}$ and $10^{5} \mathrm{eV}$.

The total count ratios of the secondary process to the total process for ionization and excitation are plotted in Fig.8. Here, it should be recalled that the secondary process means ionization or excitation process by electrons generated by the direct ionization with proton or 
hydrogen impact and in charge changing processes. Figure 8 shows that the ionization and excitation events by electron collision processes are an important share of the total processes in the higher energy region above $10^{4} \mathrm{eV}$. Total counts classified into primary and secondary processes are presented in Fig.9. The curves in Fig.9 describe the interaction processes of proton beam incident in water more precisely than the stopping cross sections in Fig.3. We illustrated the proton processes considering $\delta$-ray behavior in Fig.1. In this figure, a narrow cylinder in the central region represents the proton and hydrogen interaction area and the outer co-axial cylindrical volume depicts the spatial area in which electron tracks are spreading out. Iwanami and Oda (1999) reported on the radiological action of heavy charged particles, in which they deal with the heavy charged particle as a two-component radiation including the processes of high LET heavy ion tracks of low fluence and low LET electrons of high fluence. Our results concerning protons also illustrate the two-component nature of the radiation, which may lead to more detailed evaluation of biological effects of radiation than with the LET approach. On the one hand, if we know the accurate interaction radii "a" and "b" in Fig.1, we can estimate event densities for ionization and excitation, denoted as $\mathrm{n}_{\mathrm{K}}^{\mathrm{J}}(\mathrm{J}$ : particle name, $\mathrm{K}$ : process type), then a ratio can be defined as $\mathrm{R} \equiv \frac{\mathrm{n}_{\mathrm{ion}}^{\mathrm{e}}+\mathrm{n}_{\mathrm{exc}}^{\mathrm{e}}}{\mathrm{n}_{\mathrm{ion}}^{(\mathrm{p})}+\mathrm{n}_{\mathrm{exc}}^{(\mathrm{p})}+\mathrm{n}_{\mathrm{ion}}^{(\mathrm{H})}+\mathrm{n}_{\mathrm{exc}}^{(\mathrm{H})}}$. On the other hand, if we know the generation rates of single-strand breaks (SSB) and double-strand breaks (DSB) of a DNA chain through the ionization and excitation processes, the ratio R may be combined with the radiation weighting factor $\mathrm{w}_{\mathrm{R}}$ for protons. At least, from a physical point of view, the results in this paper support the recent recommendation of $\mathrm{w}_{\mathrm{R}}$ for protons approaching unity (ICRP-92, 2003). This tendency is attributable to the fact that the contribution of electron interaction is considerably large in the inelastic processes of protons. The natural extension of the present analysis will likely be to heavier charged particles such as carbon. 


\section{Concluding remarks}

In the present study, we have investigated ionization and excitation processes at the Bragg peak region of proton beams in liquid water. Primary processes by proton and hydrogen and secondary processes by electrons were separately treated in the estimation of ionization and excitation probabilities. The calculation results show that the contribution of secondary processes is more than $50 \%$ of the fraction of total event counts in the upper energy region of the Bragg peak and the primary ionization processes with hydrogen are dominant in the lower region. It is also shown that the production rates of ion and excited particle species are enhanced by charge changing processes between proton and hydrogen.

In addition, we have discussed the implication of primary and secondary processes suggesting a physical description of the radiation effects contrary to the conventional LET approach. 


\section{References}

Annals of the ICRP, 2003. Relative Biological Effectiveness (RBE), Quality Factor (Q), and Radiation Weighting Factor $\left(\mathrm{w}_{\mathrm{R}}\right)$. ICRP Publication 92, International Commission on Radiological Protection, Stockholm, Sweden.

Atomic Data, 1972. Energy Loss, Range, and Bremsstrahlung Yield for $10-\mathrm{keV}$ to $100-\mathrm{MeV}$ Electrons. Atomic Data 4 (1), 1-127, Academic Press, New York and London.

Champion, C., 2003. Theoretical cross sections for electron collisions in water: structure of electron tracks. Phys. Med. Biol. 48, 2147-2168

Danjo, A., and Nishimura, H., 1985. Elastic Scattering of Electrons from $\mathrm{H}_{2} \mathrm{O}$ Molecule. J. Phys. Soc. Japan 54, 1224-1227

Date, H., Ishimaru, Y., and Shimozuma, M., 2003. Electron collision processes in gaseous xenon. Nucl. Instr. and Meth. B 207, 373-380

Dingfelder, M., Hanke, D., Inokuti, M., Paretzke, H.G., 1998. Electron inelastic-scattering cross sections of liquid water. Radiat. Phys. Chem. 53, 1-8

Dingfelder, M., Inokuti, M., Paretzke, H.G., 2000. Inelastic-collision cross sections of liquid water for interactions of energetic protons. Radiat. Phys. Chem. 59, 255-275

Emfietzoglou, D., 2003. Inelastic cross-sections for electron transport in liquid water: a comparison of dielectric models. Radiat. Phys. Chem. 66, 373-385

Green, A.E.S., and Sawada, T., 1972. Ionization cross sections and secondary electron distributions. J. Atmosph. Terr. Phys. 76, 1719-1728

Grosswendt, B., and Waibel, E., 1978. TRANSPORT OF LOW ENERGY ELECTRONS IN NITROGENS AND AIR. Nucl. Instr. and Meth. 155, 145-156

International Commission on Radiation Units and Measurements, 1993. Stopping Powers and Ranges for Protons and Alpha Particles. ICRU Report 49. International Commission on Radiation Units and Measurements, Bethesda, Maryland.

International Commission on Radiation Units and Measurements, 1996. Secondary Electron Spectra from Charged Particle Interactions. ICRU Report 55. International Commission on Radiation Units and Measurements, Bethesda, Maryland.

International Atomic Energy Agency, 1995. Atomic and Molecular Data for Radiotherapy and Related Research. IAEA-TECDOC-799, IAEA, Vienna

Iwanami, S., and Oda, N., 1999. Can heavy charged particles really be regarded as high-LET radiations with respect to their radiological actions? I: LETs and fluences of heavy charged particles and associated $\delta$-rays. Phys. Med. Biol. 44, 873-886 
Miller, J.H., and Green, A.E.S., 1973. Proton energy degradation in water vapor. Radiat. Res. $54,343-363$

Olivera, G.H., Rivarola, R.D., Fainstein, P.D., 1997. LET AND w-VALUES OF WATER VAPOR UNDER ANTIPROTON IRRADIATION. Radiat. Phys. Chem. 49, 515-519

Pimblott, S.M., LaVerne, J.A., Mozumder, A. 1996. Monte Carlo Simulation of Range and Energy Deposition by Electron in Gaseous and Liquid Water. J. Phys. Chem. 100 (20), 8595-8606

Rudd, M.E., Kim, Y.-K., Madison, D.H., Gay, T.J., 1992. Electron production in proton collisions with atoms and molecules: energy distributions. Rev. Mod. Phys. 64, No.2, $441-490$

Toburen, L.H., and Wilson, W.E., 1977. Energy and angular distributions of electrons ejected from water vapor by $0.3-1.5 \mathrm{MeV}$ protons. J. Chem. Phys. 66 (11), 5202-5213

Uehara, S., Toburen, L.H., Wilson, W.E., Goodhead, D.T., Nikjoo, H., 2000. Calculations of electronic stopping cross sections for low-energy protons in water. Radiat. Phys. Chem. 59, $1-11$ 


\section{Figure captions}

Fig.1 Schematic illustration of interaction site for proton beam incident. Symbol "a" represents the radius of proton or hydrogen direct collision process reach and " $b$ " the radius of electron track process reach. Protons can be changed to hydrogen in the vicinity of the endpoint. Dots represent ionization or excitation processes.

Fig.2 Total cross sections for the different processes in liquid water: proton ionization (thick solid curve), hydrogen ionization (thick dashed curve), proton excitation (fine solid curve), hydrogen excitation (fine dashed curve), electron loss $\sigma_{01}$ (dotted curve), charge transfer $\sigma_{10}$ (dash-dotted curve), compared with ionization cross section by ICRU-49, 1993 (diamonds).

Fig.3 Stopping cross sections $\sigma_{\text {st }}$ for liquid water: proton ionization $((p)$-ion), hydrogen ionization $((\mathrm{H})$-ion), proton excitation $((\mathrm{p})$-exc), hydrogen excitation $((\mathrm{H})$-exc), charge changing (CC), and total stopping cross section, compared with ICRU-49 liquid data, 1993 (diamonds).

Fig.4 Mean numbers of ionization and excitation events in liquid water by electron incident with initial energy $E$. These are referred to as $k_{\text {ion }}(W)$ and $k_{\text {exc }}(W)$ in the text.

Fig.5 Ionization counts for different processes per $\mu \mathrm{m}$ of proton (or hydrogen) flight: proton ionization ((p)-ion), ionization by electrons arising from proton ionization ((p)-e-ion), hydrogen ionization $((\mathrm{H})$-ion), and ionization by electrons arising from hydrogen ionization ((H)-e-ion).

Fig.6 Excitation counts for the different processes per $\mu \mathrm{m}$ of proton (or hydrogen) flight: proton excitation ((p)-exc), excitation by electrons arising from proton ionization ((p)-e-exc), hydrogen excitation $((\mathrm{H})$-exc), and excitation by electrons arising from hydrogen ionization $((\mathrm{H})-\mathrm{e}-\mathrm{exc})$.

Fig.7 Ionization and excitation counts for the charge changing processes per $\mu \mathrm{m}$ of proton (or hydrogen) flight: ionization by charge transfer $((\mathrm{p} \rightarrow \mathrm{H})$-ion), ionization by electrons arising 
from electron loss process $((\mathrm{H} \rightarrow \mathrm{p})$-ion $)$, and excitation by electrons arising from electron loss process $((\mathrm{H} \rightarrow \mathrm{p})$-exc $)$.

Fig.8 Ratios of secondary and primary processes for ionization and excitation: ratio of secondary ionization to total ionization (secondary/total-ion), and ratio of secondary excitation to total excitation (secondary/total-exc).

Fig.9 Total ionization and excitation counts for the primary and secondary processes per $\mu \mathrm{m}$ of proton (or hydrogen) flight: primary ionization (primary-ion), secondary ionization (secondary-ion), primary excitation (primary-exc), secondary excitation (secondary-exc), and total count. 


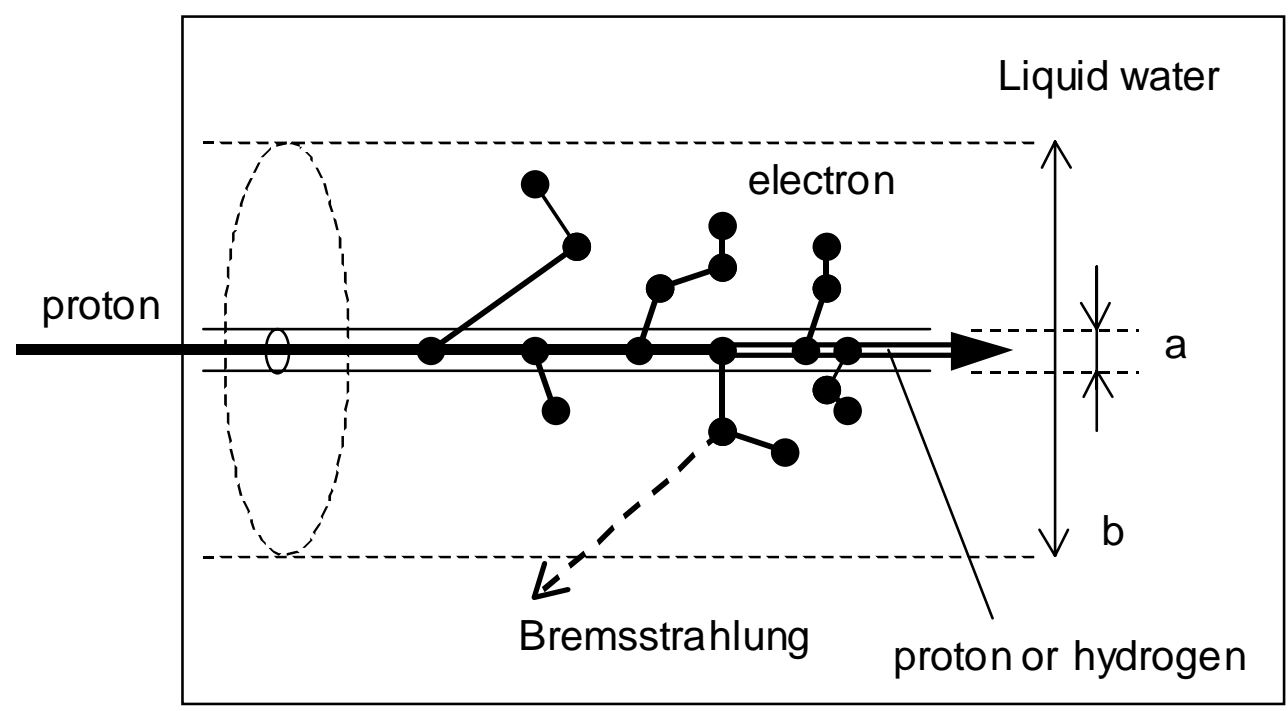

Fig.1 H. Date et al. 


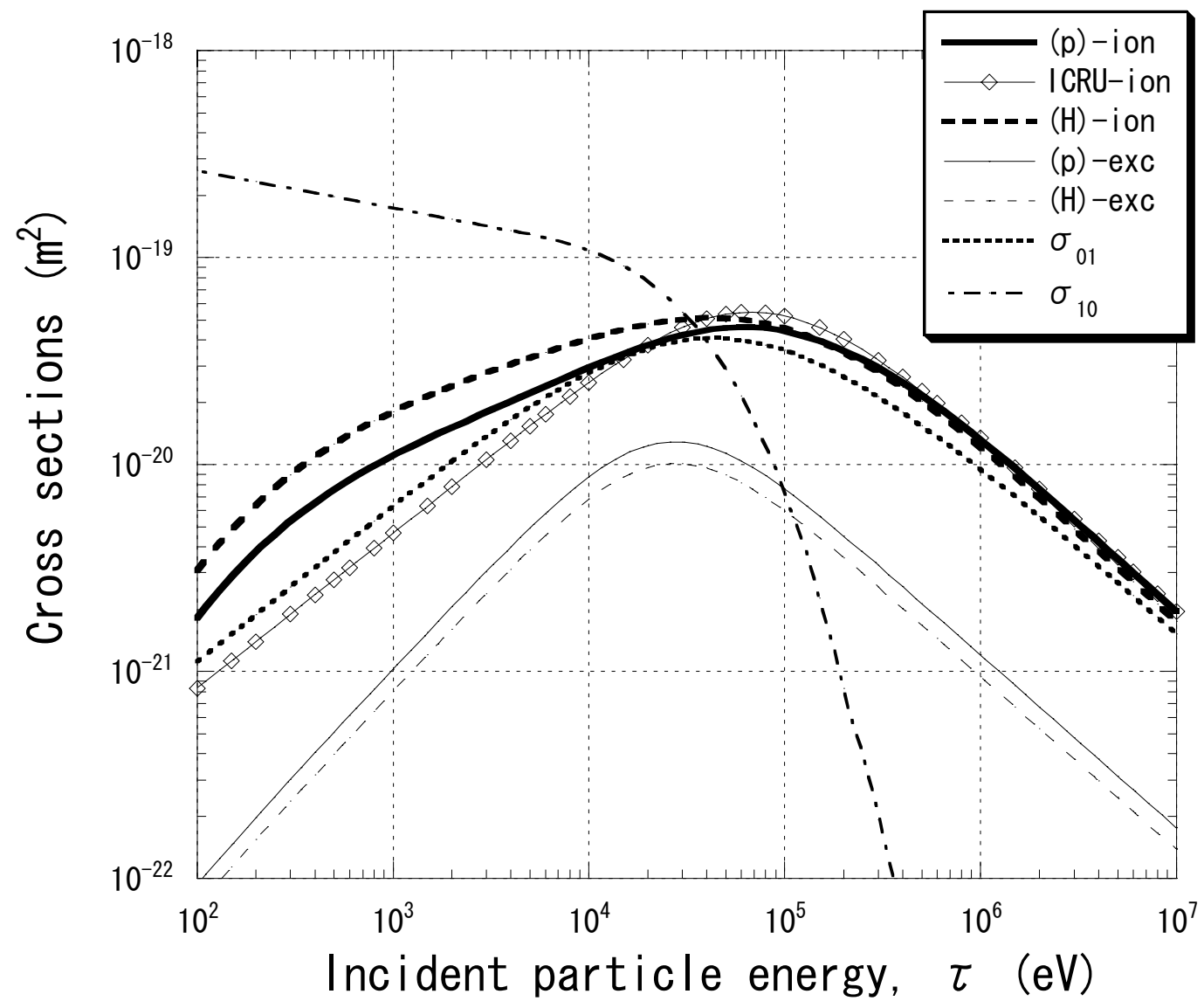

Fig. 2 H. Date et al. 


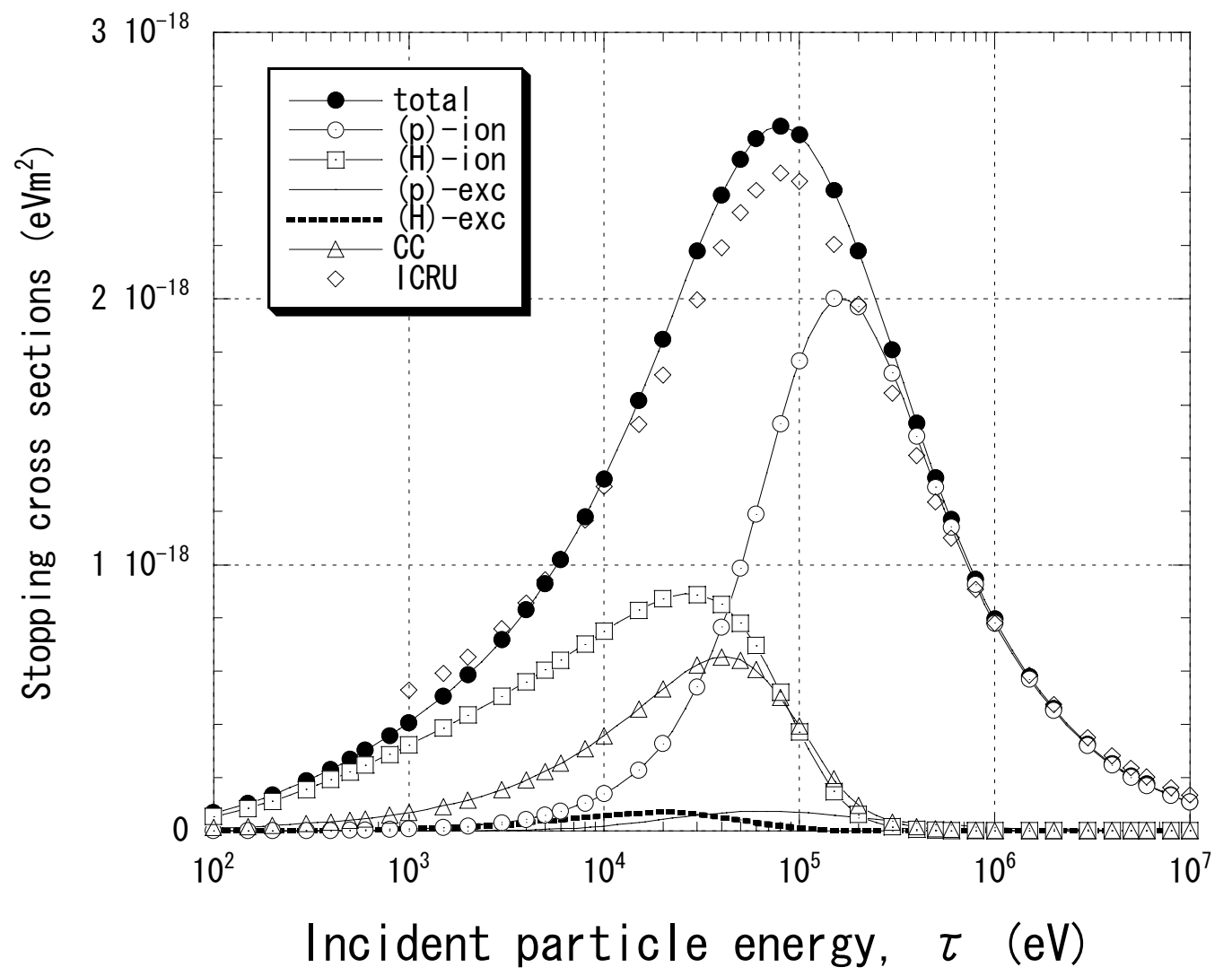

Fig.3 H. Date et al. 


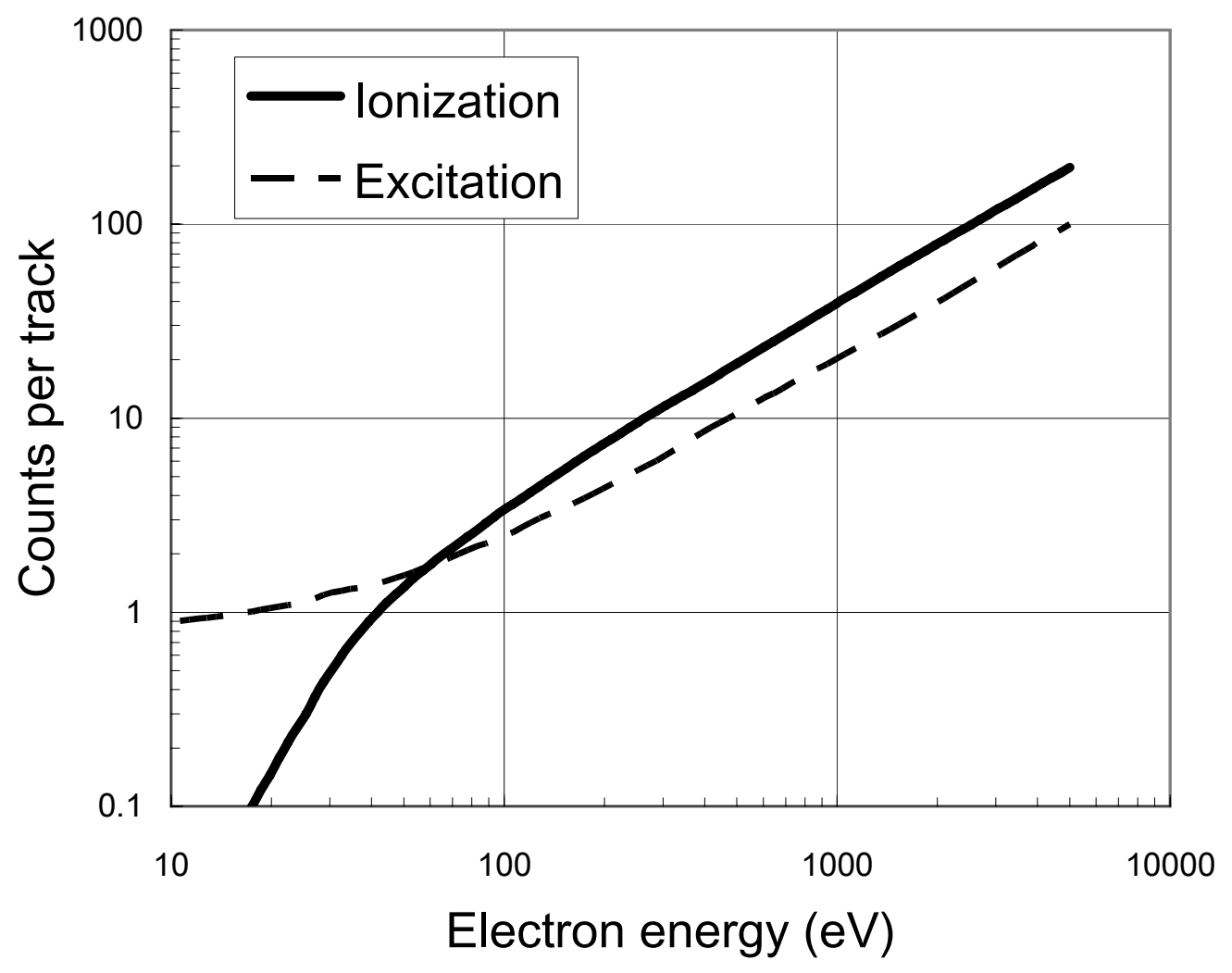

Fig.4 H. Date et al. 


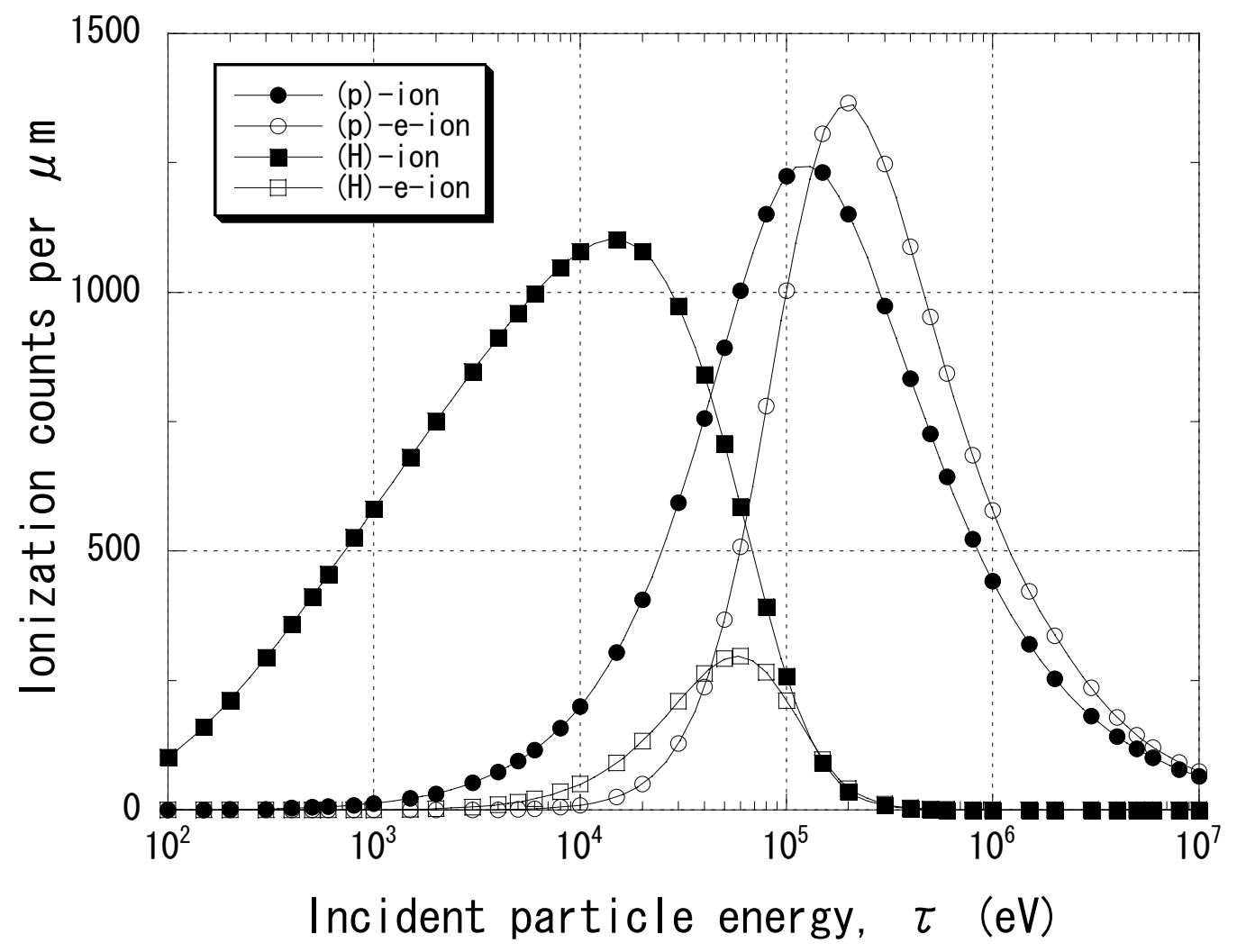

Fig.5 H. Date et al. 


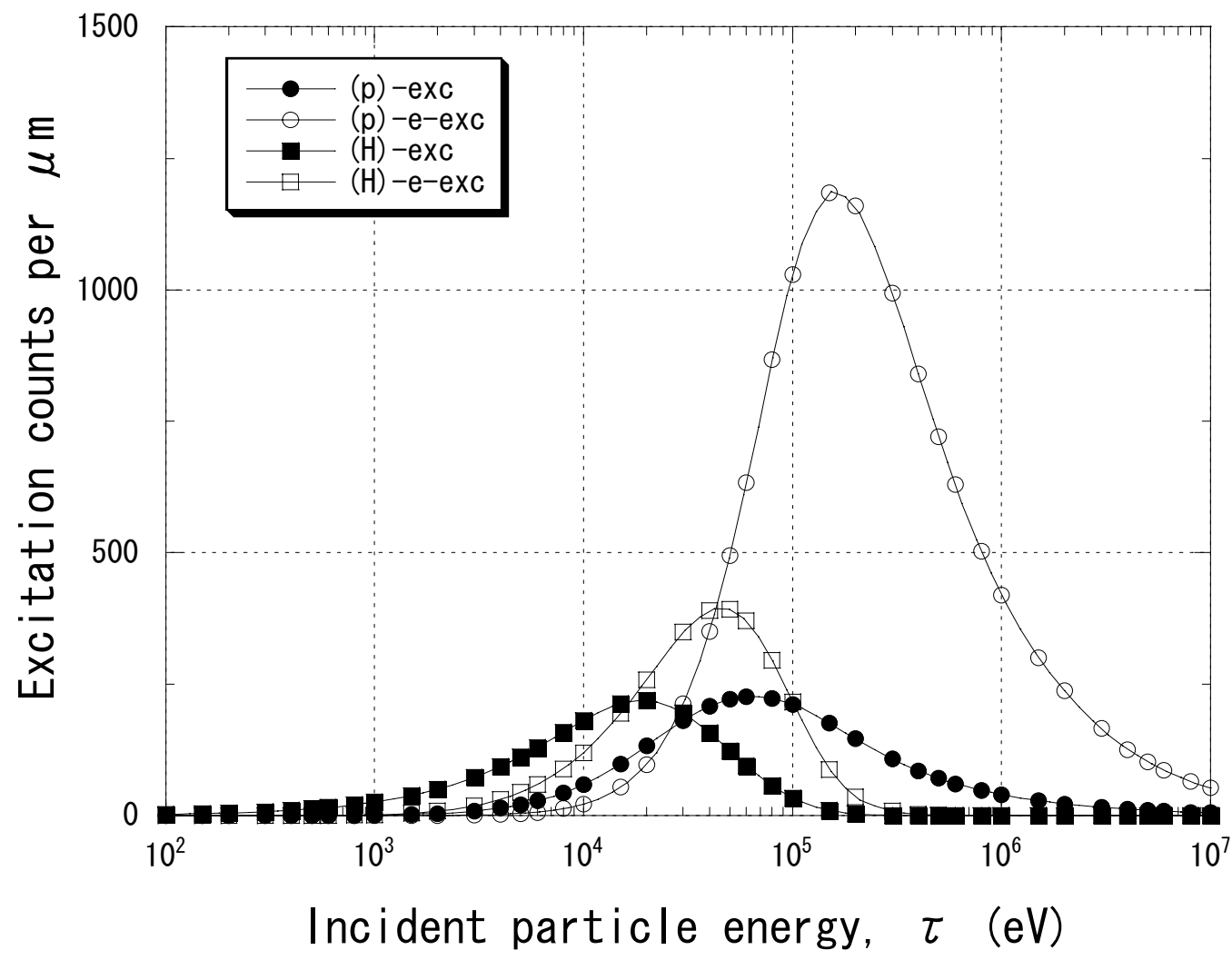

Fig.6 H. Date et al. 


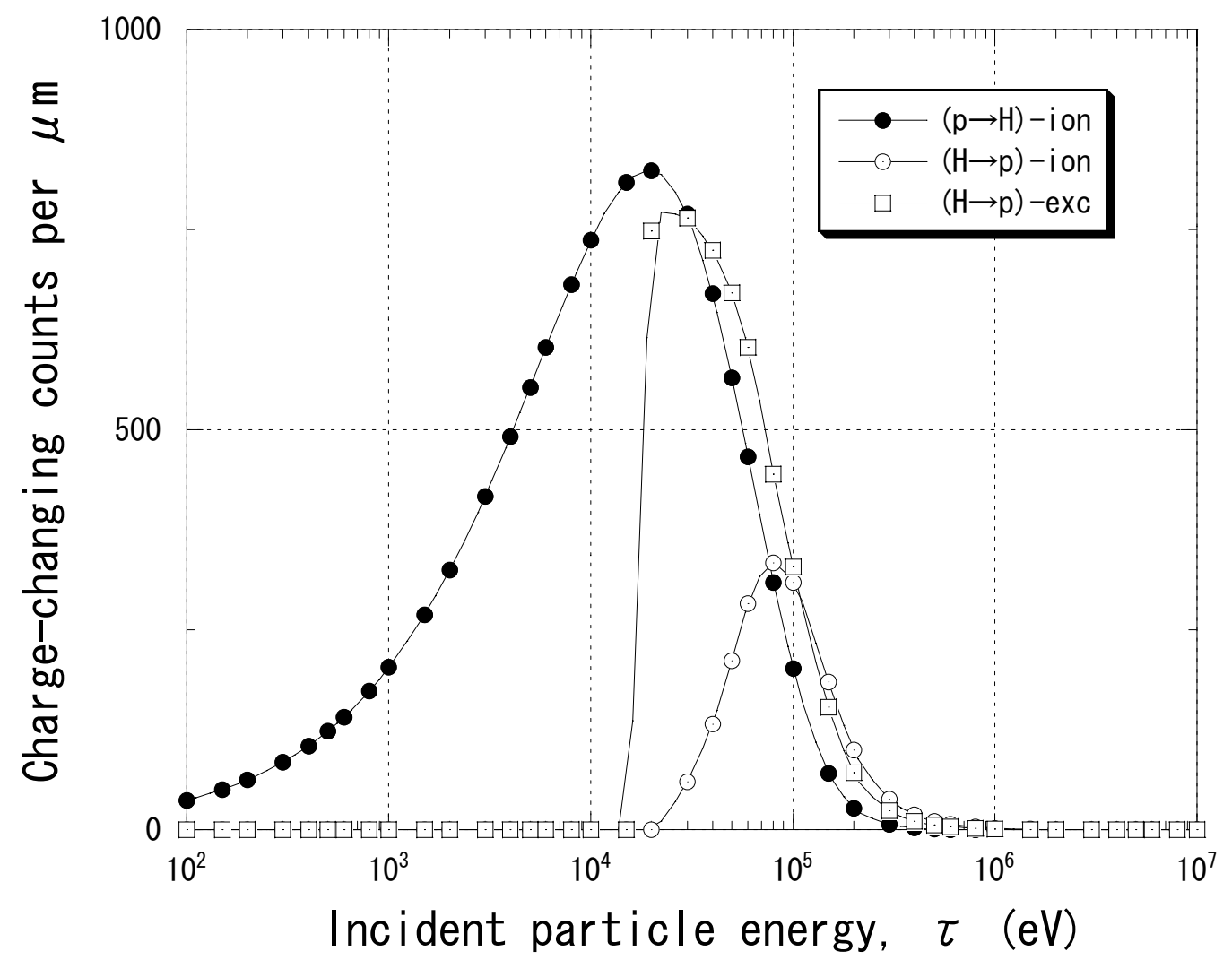

Fig.7 H. Date et al. 


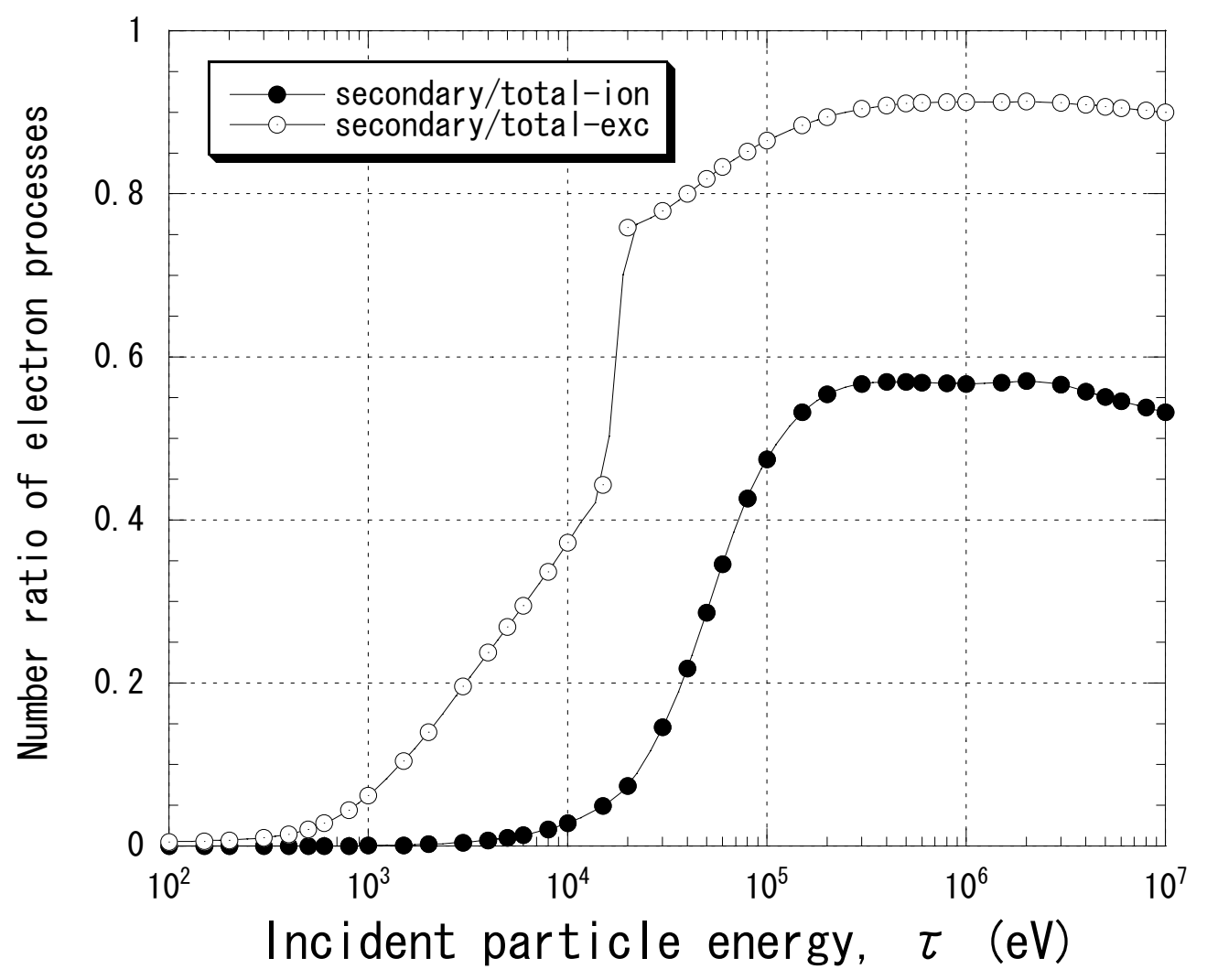

Fig. 8 H. Date et al. 


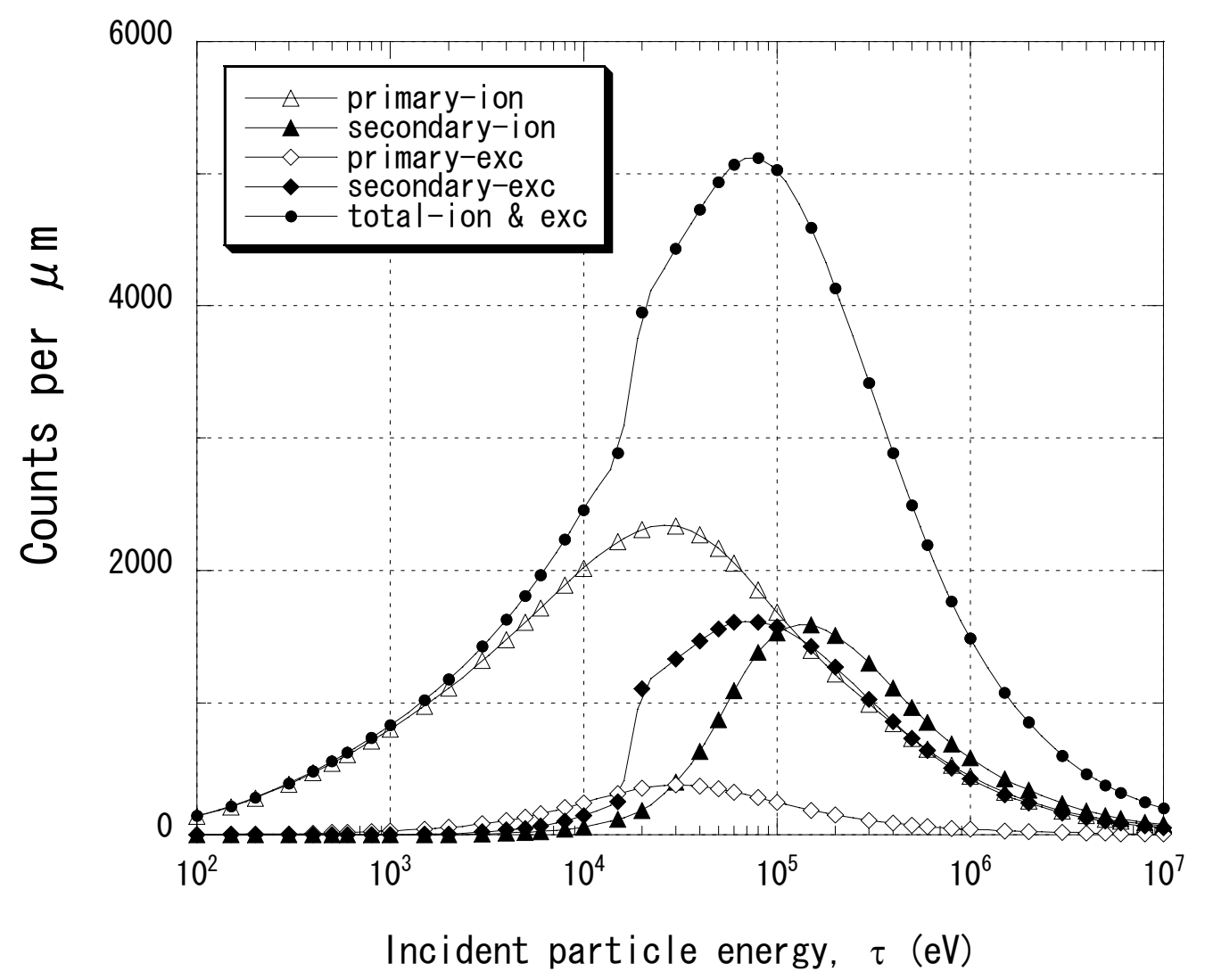

Fig.9 H. Date et al. 